\title{
Beobachtungen zum Artikelgebrauch durch Lerner des Deutschen (L1: Französisch und Italienisch)
}

\author{
Tiziana Roncoroni (Bergamo)
}

\begin{abstract}
This explorative study sheds light on article usage in German academic texts by French and Italian university (including Ph. D.) students. Given the differences between and among Romance and Germanic language systems, one might expect deviations in article usage in L2 German; in particular, speakers of Romance languages would seem likely to overgeneralise articles in their German language production. Contrary to these hypotheses, the corpora of learner varieties investigated in the present paper do not show evidence of overrepresentation and overgeneralisation of German articles, not even in generic sentences. In fact, articles tend to be underrepresented, as L2 learners often omit articles that are required in L1 German. Potential explanations of these findings are provided, giving rise to further research questions and desiderata. The latter include empirical studies on L1 German article usage in bilingual areas such as South Tirol, the use of articles in translations, and comparisons of article usage between L1 German and the L2 varieties of learners whose L1 does not have grammaticalised articles. Such studies should apply qualitative (i.e. semantic and pragmatic) as well as quantitative methods.
\end{abstract}

\section{$1 \quad$ Fragestellung}

Gegenstand des vorliegenden Artikels ist der Artikelgebrauch in deutschen akademischen Texten von fortgeschrittenen Lernern mit Italienisch und Französisch als L1.

In der Literatur wird oft und in verschiedenen Zusammenhängen auf die sprachsystemischen Unterschiede zwischen romanischen und germanischen Sprachen und innerhalb der jeweiligen Sprachzweige unter den Einzelsprachen bezüglich der zur Verfügung stehenden Artikel (Form und Funktion) hingewiesen (cf. § 2). Solche Unterschiede legen nahe, dass in der L2Produktion zu abweichenden Verwendungen der Artikel kommen kann (s. Annahme und Arbeitshypothesen in $\S 3.1$ ). Allerdings liegen bislang kaum empirische Untersuchungen in diesem Bereich vor (Ausnahmen stellen die Arbeiten von Witwicka-Iwanowska [2012] und Barton [2016] dar).

Die vorliegende Arbeit versteht sich als ein erster Schritt zur Erhellung dieses Forschungsdesiderats, indem Artikelverwendungen im konkreten Sprachgebrauch (Lernervarietät) anhand einer korpuslinguistischen Untersuchung mit Texten aus drei Korpora aufgezeigt werden. 
In $\S 2$ wird der Artikelgebrauch im Deutschen, Italienischen und Französischen verglichen, wobei auf den in der Literatur oft thematisierten Fall der generischen Referenz näher eingegangen wird (§ 2.2). Der Hauptteil der Arbeit besteht aus der empirischen Untersuchung: Zunächst werden Arbeitshypothesen und Annahmen aufgelistet, die sich von der Theorie ableiten lassen $(§ 3.1)$; im Anschluss daran werden Design und Vorgehensweise ( $\$ 3.2)$ sowie die Textgrundlage der Studie ( $\$ 3.3$ ) erläutert. Im Anschluss daran werden die Ergebnisse ausführlich präsentiert ( $\$ 3.4$ ) und ausgewertet (§ 3.5). Ausblick (§ 4) und Bibliographie schlieBen den Artikel.

\section{Artikelformen und -verwendungen im Sprachvergleich (aus theoretischer Sicht)}

Deutsch, Italienisch und Französisch sind Artikelsprachen, die über bestimmte und unbestimmte Artikel verfügen. Obwohl sie viele Ähnlichkeiten aufweisen, bestehen einige Unterschiede im Gebrauch dieser Formen. Hinzu kommen der im Italienischen, aber v. a. im Französischen hohe Stellenwert des Partitiv-Artikels sowie der häufigere Einsatz des Nullartikels und das Fehlen des unbestimmten Plural-Artikels im Deutschen.

Im Folgenden sollen die Ähnlichkeiten, aber v. a. die Unterschiede näher in Betracht gezogen werden, wobei sich das Augenmerk auf mögliche, dadurch bedingte Schwierigkeiten im deutschen L2-Artikelgebrauch richtet.

\subsection{Deutsch, Italienisch und Französisch}

Im Folgenden wird der Artikelgebrauch vergleichend erläutert; die Darstellung richtet sich hauptsächlich nach der IDS-Grammatik (Zifonun et al. 1997), z. T. der Duden-Grammatik (72005 und ${ }^{8} 2009$ ), der Grande grammatica italiana di consultazione von Renzi/Salvi/Cardinaletti ( $\left.{ }^{2} 2001\right)$ und der Grammaire méthodologique du français von Riegel/Pellat/Rioul $\left({ }^{4} 2011\right)$.

Folgende Tabelle fasst die Artikelformen (im Nominativ) im Deutschen, Italienischen und Französischen zusammen. 


\begin{tabular}{|c|c|c|c|c|}
\hline Numerus & Artikelform & Deutsch & Italienisch & Französisch \\
\hline \multirow[t]{8}{*}{ Singular } & \multirow[t]{3}{*}{ Bestimmt } & $\operatorname{der}(\mathrm{m})$ & il / lo / l' (m) & le / l' (m) \\
\hline & & $\operatorname{die}(f)$ & la / l' (f) & la / l' (f) \\
\hline & & das (n) & 1 & / \\
\hline & \multirow[t]{3}{*}{ Unbestimmt } & ein $(m)$ & uno / un (m) & un $(\mathrm{m})$ \\
\hline & & eine (f) & una / un' (f) & une (f) \\
\hline & & ein (n) & / & / \\
\hline & \multirow[t]{2}{*}{ Partitiv } & I & $\begin{array}{l}\text { del / dello / dell' } \\
\text { (m) }\end{array}$ & du / de l' (m) \\
\hline & & I & della / dell' (f) & de la / de l' (f) \\
\hline \multirow[t]{5}{*}{ Plural } & \multirow[t]{3}{*}{ Bestimmt } & $\operatorname{die}(\mathrm{m})$ & i / gli (m) & les (m) \\
\hline & & $\operatorname{die}(f)$ & le (f) & les (f) \\
\hline & & die (n) & / & / \\
\hline & \multirow[t]{2}{*}{ Unbestimmt } & I & dei / degli (m) & $\operatorname{des}(\mathrm{m})$ \\
\hline & & I & delle (f) & $\operatorname{des}(f)$ \\
\hline
\end{tabular}

Tabelle 1: Die Artikelformen im Deutschen, Italienischen und Französischen ${ }^{1}$

Die semantischen Funktionen der Artikel sind:

(i) in dem durch Appelativum bzw. pluralische oder Substanz-NP [= Nominalphrase] definierten Bereich ein definites Denotat für das Argument einer Prädikation zu bestimmen; die Markierung der Definitheit leisten die Formen des definiten [= bestimmten] Artikels;

(ii) festzulegen, daß in dem durch ein Appellativum im Singular oder eine Substanz-NP im Singular vorgegebenen Bereich (mindestens) ein Gegenstand ist, auf den die Prädikation zutrifft; dies leisten die Formen des indefiniten [= unbestimmten] Artikels, auch bloße [= artikellose] Substanzausdrücke und bloße Plurale.

(Zifonun et al. 1997: 1958)

Der unbestimmte Artikel dient dazu, „einen Gegenstand in einen Verwendungszusammenhang allererst einzuführen, ihn zu konstituieren, dem Adressaten bekannt zu machen“ (ibd.: 34). Durch den unbestimmten Artikel wird ein beliebiges Denotat als Gegenstand der Prädikation aufgegriffen (die Bestimmtheit des Referenten wird dabei nicht thematisiert, cf. BisleMüller 1991: 50). Durch den bestimmten Artikel wird hingegen das salienteste Denotat bezeichnet (cf. Zifonun et al. 1997: 2059-2060). Das Einvernehmen mit dem Hörer über den Referenten wird problemlos vorausgesetzt (cf. Bisle-Müller 1991: 100); diese deutliche Referenz-Zuweisung ist dadurch gegeben, dass der bestimmte Artikel die Identifizierbarkeit des Referenten, die Gesamtheit des Referenten (d. h.: der Referent wird insgesamt von dem besprochenen Geschehen erfasst) oder die Einzigkeit des Referenten gewährleistet (cf. Witwicka-Iwanowska 2012: 136-137). ${ }^{2}$

\footnotetext{
${ }^{1}$ Der Partitivartikel wird in mehreren Grammatiken der romanischen Sprachen als Variante des unbestimmten Artikels aufgefasst (cf. Renzi/Salvi/Cardinaletti ${ }^{2} 2001: 377$ ).

2 Die deutschen Grammatiken gehen der Frage der Identifizierung des Referenten sehr genau nach. Laut der Duden-Grammatik ist der Referent aufgrund der bereits erfolgten Einführung (Vorerwähnung, Vorinformation), einer Attribuierung im vorliegenden Ausdruck, seines bekannten Charakters (innerhalb des Adressaten-Kreises) oder seiner Einmaligkeit hinreichend identifiziert (cf. Duden 2005: 299-302). Helbig/Buscha ( 2005 : 336) führen die Identifizierung des Referenten der NP mit dem bestimmten Artikel auf folgende Faktoren zurück: Individualisierung, Situationskontext, sprachlichen Kontext (bei Anaphern) und Generalisierung. Zifonun et al. (1997: 34) zufolge wird der bestimmte Artikel bei Referenten eingesetzt, die aus einem situativ oder textuell vorgege-
} 
Was die Gesamtverwendung der Artikel betrifft, ${ }^{3}$ ist zunächst festzuhalten, dass in den romanischen Sprachen die Tendenz zum obligatorischen Artikelgebrauch (oder zum Gebrauch von anderen Determinativen) besteht. Unter den romanischen Sprachen ist das Französische diejenige, die NPs ohne Determinative am wenigsten ermöglicht. ${ }^{4}$ Der Einteilung von Longobardi (2001) zufolge zählt Französisch zu den indogermanischen Sprachen, die keine NPs ohne Artikel aufweisen.

Der Artikel in italienischen und französischen NPs fehlt nur unter besonderen Bedingungen:

- $\quad$ bei bürokratischem und sehr formalem Stil (in präverbalen Plural-NPs, die durch Adjektive oder Relativsätze modifiziert werden, wie in „Eventuali assenze saranno segnalate per tempo“) (cf. Renzi/Salvi/Cardinaletti ${ }^{2} 2001$ : 389-390);

- $\quad$ in Ausrufen (cf. Renzi/Salvi/Cardinaletti ${ }^{2} 2001:$ 420), Aufzählungen, Abkürzungen, Telegrammen, Notizen, Titeln bzw. Beschriftungen (cf. Renzi/Salvi/Cardinaletti ${ }^{2} 2001$ : 414-415 und Riegel/Pellat/Rioul ${ }^{4} 2011:$ 310-311);

- in koordinierten NPs, die den gleichen Artikel haben (wie in „le colline e _ montagne del Piemonte“ vs. „*i colli e _ montagne del Piemonte“) (cf. Renzi/Salvi/Cardinaletti ${ }^{2} 2001$ : 432-433 und Riegel/Pellat/Rioul ${ }^{4} 2011$ : 310 für ähnliche Beispiele im Französischen);

- bei geschlossenen Paaren oder Serien und totalisierenden Zusammensetzungen (wie in it. „genitori e figli““ und fr. ,parents et enfants“) (cf. Renzi/Salvi/Cardinaletti ${ }^{2} 2001: 403$ und Riegel/Pellat/Rioul ${ }^{4} 2011:$ 309);

- bei Eigennamen von Städten und Personen (wie in it. „Thomas è sportivo“ und fr. „Thomas est sportif“); Toponyme kommen hingegen meist mit dem bestimmten Artikel vor (cf. Riegel/Pellat/Rioul ${ }^{4} 2011: 315$ );

- $\quad$ bei adjektivlosen Prädikativa, wie bspw. bei der Bezeichnung des Berufs (wie in it. „Ѐ medico“ und fr. „Il est médecin“) und bei der Angabe von Nationalität (wie in it. „È italiana“ und fr. „Elle est italienne“) und Weltanschauung (wie in it. „Sono mussulmano“ und fr. ,Je suis musulman“) ${ }^{5}$ sowie in Appositionen (cf. Renzi/Salvi/Cardinaletti ${ }^{2} 2001$ : 414 419 und Stollhans 2012: 611-612);

\footnotetext{
benen Bereich herausgegriffen werden. Die Präsupposition der Einzigkeit ist also in dem Kontext gegeben; ist es nicht der Fall, wird die NP auf den in dem jeweiligen Kontext hervorstechendsten (salientesten) Referenten bezogen (cf. Zifonun et al. 1997: 1958).

${ }^{3}$ Im Rahmen der vorliegenden Studie wird nur der freie Gebrauch der Artikel erläutert, der von dem jeweiligen Zusammenhang bestimmt wird (cf. Duden 2005: 301). Auf den sekundären Artikelgebrauch (d. h. den Artikelgebrauch, der nur unter bestimmten Umständen und in bestimmten Gebrauchsweisen von den Grundregeln abweicht) wird hier nicht eingegangen. Das Gleiche gilt für den gebunden Artikelgebrauch, der beispielsweise feste Sätze und idiomatische Ausdrücke charakterisiert (für das Italienische cf. hierzu Renzi/Salvi/Cardinaletti ${ }^{2}$ 2001: 427).

${ }^{4}$ Man denke bspw. an Verwendungen des bestimmten Artikels im Französischen vs. des Nullartikels im Italienischen wie bei Anreden (wie in it. ,signor presidente,..." vs. fr. ,,monsieur le président,...", it. „,arrivederci amici“ vs. fr. „au revoir les amis“) (cf. Stollhans 2012: 612) und bei absoluten Superlativformen (wie in it. „l'amico migliore“ vs. fr. „l'ami le meilleure“); im Italienischen wären Phrasen wie „il motivo il più cogente“ literarisch markiert (Renzi/Salvi/Cardinaletti ${ }^{2}$ 2001: 376).

${ }^{5}$ In deutschen Grammatiken spricht man von Funktionsbezeichnungen im weiteren Sinne, der größten Untergruppe prädikativer NPs im klassifizierenden Gebrauch (cf. schon Brinkmann 1962: 59). Werden NPs hingegen beschreibend verwendet, kommen sie mit einem Adjektiv und/oder dem unbestimmten Artikel vor.
} 
- $\quad$ in bestimmten Angaben, meist Präpositionalphrasen; ${ }^{6}$

- $\quad$ bei einigen Fällen der Generizität (cf. § 2.2).

Kommt eine Spezifizierung dieser NPs oder der beinhalteten Substantive hinzu, wird der Artikelgebrauch obligatorisch (cf. Renzi/Salvi/Cardinaletti ${ }^{2} 2001: 410$ und Riegel/Pellat/Rioul ${ }^{4} 2011$ : 317); ein Beispiel ist „È medico“ vs. „È un bravo medico“.

Hingegen sind in den germanischen Sprachen (dem Englischen in erster Linie, aber auch dem Deutschen) nicht nur in den o. g. Fällen, sondern viel häufiger NPs mit dem Nullartikel/mit anderen Determinativen belegt.

Einen großen Bereich stellen die Kombinationen mit Eigennamen dar: Die meisten Ländernamen (Neutra), Ortsnamen, Vornamen auch mit Herr, Frau und Fräulein (cf. Zifonun 1997: 1931-1933), ein Teil der Gebiets- und Ländernamen, viele Namen von Firmen und Institutionen und viele Festtage erscheinen im Hochdeutschen mit dem Nullartikel (cf. Duden 2005: 307-308). Nur die übrigen Eigennamen treten mit dem bestimmten Artikel auf (cf. Duden 2005: 308).

Zweitens sind auf der einen Seite die artikellosen Plural-NPs, die im Singular den unbestimmten Artikel hätten (der fehlende Artikel geht auf die Tatsache zurück, dass das Deutsche über keine unbestimmten Pluralartikel verfügt), und auf der anderen Seite die Singular-NPs, deren Substantive nicht zählbar sind ${ }^{7}$ (cf. Duden 2005: 338), zu nennen. Im Italienischen und im Französischen wird in diesen Fällen meist der partitive Artikel benutzt: On a de la farine und Abbiamo della farina vs. Wir haben Mehl.

Drittens sind Kollektiva zu erwähnen (wie in „Der Wald besteht aus Bäumen“), deren Größe nicht weiter spezifiziert wird (cf. Zifonun et al. 1997: 1953) und in deren Semantik kumulative Denotation vorliegt (cf. ibd.: 1954).

Eine vierte große Gruppe stellen generische Ausdrücke dar: Das Deutsche setzt üblicherweise den Nullartikel ein, obwohl der bestimmte Artikel auch möglich ist (cf. § 2.2 und die Ergebnisse der empirischen Untersuchung von Barton et al. 2015). ${ }^{8}$ Der Duden-Grammatik zufolge bleibt der Nullartikel insgesamt die präferierte und unmarkierte Wahl (cf. Duden 2009: 296).

In den romanischen Sprachen, und besonders systematisch im Französischen, muss in generischen NPs der bestimmte Artikel vorhanden sein (cf. Barton et al 2015: 148):

(1) Fr.: Les chats ont quattre pattes. *Chats ont quattre pattes.

It.: I gatti hanno quattro zampe. *Gatti hanno quattro zampe.

Dt.: (Die) Katzen haben vier Pfoten. ${ }^{9}$

\footnotetext{
${ }^{6}$ Präpositionalphrasen mit $d i$ mit folgenden Funktionen: Thema, Stoff, Partitiv, Qualität. Beispiele: „un bicchiere di vino“ und ,il libro di storia“ (cf. Renzi/Salvi/Cardinaletti ${ }^{2} 2001$ : 422-423). Präpositionalphrasen mit tra, con/senza wie „con gentilezza“ und a/in in Ortsangaben wie ,a tavola“ und „in spiaggia“ (cf. Renzi/Salvi/Cardinaletti ${ }^{2}$ 2001: 421-423). Für Beispiele für französische prädikative Ausdrücke (wie „Il y a attente“) und französische Präpositionalphrasen ohne Artikel s. Riegel/Pellat/Rioul ( ${ }^{4} 2011:$ 310-314).

${ }^{7}$ In erster Linie Substanznomina (cf. Zifonun et al. 1997: 1955), auch in der Behälterkonstruktion wie in „ein Glas Bier“" (cf. Zifonun et al. 1997: 1979, 1985).

${ }^{8}$ Witwicka-Iwanowska (2012: 144) kommt allerdings zu dem Schluss, dass die generische Lesart bei artikellosen NPs nicht typisch ist.

${ }^{9}$ Beispiele z. T. aus Barton (2016: 56).
} 
Die Deutung der Sätze als generisch oder spezifisch ist dann dem jeweiligen pragmatischen Kontext überlassen. Das Gleiche gilt für die deutsche Version mit dem bestimmten Artikel.

Die Artikel-Grammatikalisierung in den romanischen Sprachen ist insgesamt besonders fortgeschritten; der bestimmte Artikel kommt mit wenigen Ausnahmen (s. oben) auch bei Eigennamen vor und wird somit auch nicht referenziell verwendet (cf. Lyon 1999 und Barton 2016: 64). Im Italienischen begleitet der bestimmte Artikel mit wenigen Ausnahmen (Verwandtschaftsbezeichnungen im Singular außer mit loro) selbst NPs, die ein Possessivadjektiv aufweisen (cf. Renzi/Salvi/Cardinaletti ${ }^{2} 2001$ : 374 und Barton et al. 2015: 149):

(2) It.: La mia casa.

Fr.: Ma maison; *La/une ma maison.

Dt.: Mein Haus; *Das/ein mein Haus.

Dies ist sogar bei Körperteilen der Fall (wie in it. „la mia mano“), bei denen selbst beim Ausfallen des Possessivadjektivs die possessive Lesart präferiert wird (cf. Barton 2016: 65-66). In diesem Fall spricht man von unveräußerlicher Zugehörigkeit wie in „mi fa male la testa“ (cf. Renzi/Salvi/Cardinaletti ${ }^{2}$ 2001: 400). ${ }^{10}$

Darüber hinaus kommt der bestimmte Artikel in romanischen Sprachen bei der veräußerlichen Zugehörigkeit (z. B. ,abbiamo fatto le valigie“), in anaphorischen und kataphorischen Ausdrücken, bei geteiltem Wissen sowie in Ausdrücken mit einem einmaligen Referenten (wie ,il sole“) vor (cf. Renzi/Salvi/Cardinaletti ${ }^{2}$ 2001: 397-401).

Der deutsche bestimmte Artikel hat wiederum andere Funktionen; er dient bspw. nicht nur zur Enkodierung der Definitheit, sondern auch, wenn betont, als deiktisches Determinativ in der gesprochenen Sprache: „Der Platz ist besetzt“ (cf. Zifonun et al. 1997: 37, 1960 sowie Gunkel 2006: 82); 11 die Duden-Grammatik spricht in diesem Zusammenhang von einer „Übergangszone zur zeigenden (deiktischen) Verwendung und damit zum demonstrativen Artikelwort“" (Duden 2005: 300). Die anderen üblichen Funktionen unterscheiden sich nicht von denen des bestimmten Artikels in den romanischen Sprachen.

Der deutsche unbestimmte Artikel kommt außer in exemplarischen Generalisierungen (cf. § 2.2) nur in NPs vor, deren Substantive im Singular stehen, zählbar sind und von keinem anderen Artikel (d.h. bestimmtem Artikel oder Nullartikel) begleitet werden (cf. Duden 2005: 337). Auch in den romanischen Sprachen markiert der unbestimmte Artikel den Verweis auf Elemente, die auf keine andere Weise determiniert werden (cf. Riegel/Pellat/Rioul ${ }^{4} 2011$ : 293), und erfüllt somit eine (tatsächlich oder virtuell) existentielle Funktion (wenn der semantische Inhalt mit „il y a/c'è/ci sono“ paraphrasiert werden kann) oder eine partitive Funktion (bei unzählbaren Substantiven und Abstrakta wie in ,de la farine“ und ,du courage“) (cf. Riegel/Pellat/Rioul ${ }^{4} 2011$ : 293-296). Im Vergleich zum Deutschen ist die Verbreitung des unbestimmten Artikels in den romanischen Sprachen deutlich größer, da es auch Pluralformen gibt.

\footnotetext{
${ }^{10}$ Das Französische verhält sich auf eine ähnliche Weise: „Il s'est lavé les mains“ (cf. Riegel/Pellat/Rioul ${ }^{4} 2011$ : 285).

${ }^{11}$ In der Tat stammt der bestimmte Artikel aus dem Demonstrativpronomen, das im Grammatikalisierungsprozess an Bedeutung und Betonung verloren hat.
}

ISSN 1615-3014 


\subsection{Generizität}

An dieser Stelle gilt es, auf die verschiedenen Fälle der Generizität näher einzugehen, wobei weitere Unterschiede im Artikelgebrauch herauszuarbeiten sind.

Die Beispiele aus der IDS-Grammatik für Sätze, die als generisch verwendet werden können, sind die folgenden (Zifonun et al. 1997: 2055; Hinzufügung der Bezeichnungen, TR):

(3) Eine Schwalbe macht noch keinen Sommer.

Der Schwabe ist fleißig.

Katzen würden Whiskas kaufen.

Die Mongolen waren geschickte Reiter.

Löwen haben eine Mähne.

Ein Löwe hat eine Mähne.

$\rightarrow$ abgeleitete Gattungsprädikat

$\rightarrow$ abgeleitete Gattungsprädikat

(Der/Ein Läufer zieht diagonale. [bei Schach]

Läufer ziehen diagonal [bei Schach]

Der Löwe ist eine Raubkatze.

Der Saurier ist ausgestorben.

Die Saurier sind ausgestorben.

$\rightarrow$ gesetzartige Aussagen)

$\rightarrow$ Gattungsprädikat

$\rightarrow$ Gattungsprädikat

$\rightarrow$ Gattungsprädikat

Die Beispiele belegen, dass alle Artikelformen in den NPs generischer Sätze möglich sind (cf. auch Stollhans 2012: 614).

Die NPs, die Gattungsprädikate denotieren, weisen den bestimmten Artikel (aber auch Eigennamen) auf. Gattungsprädikate sind diejenigen, die der ganzen Gattung zugesprochen werden müssen. Der unbestimmte Artikel und der Nullartikel (bei Pluralnomen) kommen in NPs vor, die abgeleitete Gattungsprädikate denotieren, d. h. denjenigen, die über typische Vertreter der Gattung prädizieren (cf. Zifonun et al. 1997: 2056). Auch der Duden-Grammatik (2005: 304) zufolge dienen die Versionen mit dem unbestimmten Artikel und dem Nullartikel zur Enkodierung einer exemplarischen Generalisierung (man hat ein typisches Exemplar der betroffenen Gattung vor Augen).

Die Interpretation der NPs ist für die generische Interpretation der Sätze von wesentlicher Bedeutung (cf. Zifonun et al. 1997: 2059). Sie kommt folgendermaßen zu Stande: Ist im Falle einer NP mit bestimmtem Artikel das salienteste Denotat nicht zu erkennen, wird die NP als Gattungsbezeichnung interpretiert (cf. Zifonun 1997: 2060). Ist im Falle einer NP mit dem unbestimmten Artikel oder dem Nullartikel die Auswahl eines geeigneten Bezuges nicht möglich, wird die generische Lesart vorgezogen (cf. Zifonun 1997: 2060, 2062).

Krifka et al. (1995) arbeiten die Unterscheidung zwischen den o. g. generischen Sätzen (,,characterizing sentences“) und generischen NPs an sich (Gattungs-NPs = ,kind-referring NPs“) wie in „The potato was first cultivated in South America“ (Krifka s. a.: 1) heraus. ${ }^{12}$ Letztere bringen tendenziell den bestimmten Artikel mit sich (cf. auch Barton et al. 2015; Brugger 1993; Duden 2009: 296 und Stollhans 2012: 614); erstere geben allgemeine Gattungseigenschaften wieder und gehen wie oben gezeigt mit mehreren Artikelformen einher.

\footnotetext{
12 Die Einteilung ist nicht immer einfach. Krifka et al. (1995) bringen selber Beispiele von Sätzen, die an sich bereits generisch sind und gleichzeitig eine generische NP beinhalten, wie das Beispiel „The potato is highly digestive" (cf. auch Krifka s. a.: 1 und Barton 2016: 71).
} 
Generische Sätze, die Eigenschaften einzelner Mitglieder einer Gattung zum Ausdruck bringen (abgeleitete Gattungsprädikate, auch Sätze mit „,individual-level“ Prädikaten genannt), werden auch in Grammatiken der romanischen Sprachen von denen unterschieden, die sich nicht auf ein einzelnes Mitglied der Spezies beziehen können (Sätzen mit „kind-level“ Prädikaten, Gattungsprädikaten) (cf. Riegel/Pellat/Rioul ${ }^{4}$ 2011: 283). Der bestimmte Artikel bleibt in NPs beider Untergruppen allerdings die deutlich bevorzugte Option (cf. Zamparelli 2002: 3; Stollhans 2012 und Barton 2016: 83, 85); das, was ausfällt, ist die o. g. zweideutige Deutung des bestimmten Artikels (generisch vs. spezifisch): Es bleibt nur die generische Lesart. Der bestimmte Artikel im Singular kennzeichnet die Referenz auf die gesamte Gattung, während der bestimmte Artikel im Plural die kognitive Assoziation von einigen Individuen hervorruft (cf. Renzi/Salvi/Cardinaletti ${ }^{2} 2001:$ 378). ${ }^{13}$ Andere Artikel-Optionen sind marginal. Wird der unbestimmte Artikel verwendet, muss das Prädikat Merkmale einer Gattung zum Ausdruck bringen, damit das Exemplar in der NP als typisch für seine Gattung gedeutet wird (cf. Riegel/Pellat/Rioul ${ }^{4}$ 2011: 295 für das Französische). ${ }^{14}$ Der Nullartikel kommt nur in einigen Fällen im Plural und selten im Singular und lediglich als Alternative in Frage (cf. Renzi/Salvi/Cardinaletti ${ }^{2}$ 2001: 388-390, 392).

Im Sprachvergleich lässt sich also Folgendes festhalten (cf. z. T. Stollhans 2012: 616):

- In generischen Singular-NPs wird im Deutschen meist der bestimmte Artikel verwendet; in den romanischen Sprachen ist er die einzige Möglichkeit.

- In Sätzen mit „kind-level“ Prädikaten präferiert das Deutsche den bestimmten Artikel, wobei der Nullartikel nicht ausgeschlossen ist. Das Französische und das Italienische verlangen den bestimmten Artikel.

- In Sätzen mit ,individual-level“ Prädikaten präferiert das Deutsche den Nullartikel. Wiederum steht im Französischen und im Italienischen nur der bestimmte Artikel.

- $\quad$ Hinzu kommt der Fall der generischen Objekt-NP, die meist von statischen Verben des (nicht)-Mögens regiert wird, wie in:

(4) Dt.: Ich mag Kaffe aber hasse Süßigkeiten.

Fr.: J'aime le café, mais je déteste les gâteaux.

It.: $\quad$ Mi piace il caffè, ma odio i dolci.

Im Gegensatz zum deutschen Nullartikel benötigen die romanischen Sprachen auch hier den bestimmten Artikel (allerdings ist der Nullartikel im Italienischen, wie bspw. in Mangio car$n e$, und der Partitivartikel im Französischen, wie bspw. in Je mange de la viande, möglich).

Die bisher einzige kontrastive empirische Untersuchung (die Dissertation von Barton 2016), mit dem deutsch-französischen Artikelgebrauch in Plural-Subjekt-NPs generischer Ausdrücke als Schwerpunkt, untermauert diese Schlussfolgerungen. ${ }^{15}$ Französische Muttersprachler er-

\footnotetext{
${ }^{13}$ Riegel/Pellat/Rioul ( $\left.{ }^{4} 2011: 285\right)$ sprechen für das Französische allerdings von einer weniger vollständigen Generizität im Fall der Singular-NPs.

${ }^{14}$ Renzi/Salvi/Cardinaletti ( $\left.{ }^{2} 2001: 386-387\right)$ bringen für das Italienische folgendes Beispiel: „Un bambino coraggioso non piange“ bzw. „Dei bambini coraggiosi non piangono“.

15 Obwohl der bestimmte Artikel in solchen Kontexten akzeptabel ist, wird er in $50 \%$ der Fälle durch den Nullartikel korrigiert; der Nullartikel wird hingegen immer akzeptiert; in Fällen, in denen beide möglich sind, geht der bestimmte Artikel überwiegend mit der spezifischen, der Nullartikel mit der generischen Lesart einher
} 
setzen sogar den grammatisch korrekten bestimmten Artikel durch stärker definite Determinative wie Demonstrativa oder Possessiva (cf. Barton 2016: 176).

Bezüglich der Generizität werden weitere theoretische Fragestellungen behandelt, v. a. zur Deutung und zur Semantik von NPs mit dem Nullartikel (cf. Krifka et al. 1995 und 2004 vs. Chierchia 1998; cf. auch Longobardi 1994 und Zamparelli 2002). Ein anderer Schwerpunkt liegt auf der Unterscheidung und Kategorisierung von generischen Ausdrücken auf der Suche nach der Quelle der generischen Interpretation, die in der Struktur der Subjekt-NP, im Kontext oder in der Prädikatsbedeutung liegen kann (cf. Barton et al. 2015). Als Basis dieser Diskussion gilt das Grundwerk The generic book (Carlson/Pellier 1995), das mehrere Beiträge zum Rahmenthema der Quelle(n) der generischen Deutung in kognitiver, logischer, ontologischer und lexikalischer Perspektive sammelt. Im Rahmen der vorliegenden Arbeit wird nicht näher darauf eingegangen.

\section{Empirische Untersuchung}

Ausgehend von den sprachsystemischen Unterschieden, die in Deutsch, Italienisch und Französisch bestehen (cf. § 2), stellen sich Fragen, die für den DaF-Unterricht von Belang sind:

- Lassen sich typische Abweichungsmuster im deutschen L2-Artikelgebrauch feststellen? Welche?

- Könnten Merkmale des Artikelgebrauchs in der L1 die Quelle davon sein?

Gerade um die zweite Frage zu beantworten, müssen vorab einige, aus der Theorie abgeleitete Arbeitshypothesen und Annahmen formuliert werden.

\subsection{Arbeitshypothesen und Annahmen}

Die kontrastive theoretische Darstellung des Artikelgebrauchs (cf. § 2) lässt annehmen,

1) dass italienische und v. a. französische Lerner des Deutschen in ihren deutschen L2-Texten den bestimmten Artikel überdurchschnittlich verwenden können, nämlich auch bei den Fällen der generischen Referenz, die im Deutschen eher durch den Nullartikel enkodiert werden. Gleiche Annahmen finden sich oft in der Literatur, die sich mit generischen Ausdrücken im Sprachvergleich befasst (cf. Stollhans 2012: 617, der von „overuse des bestimmten Artikels“ spricht).

2) Nicht nur im Bezug auf die Generizität, sondern im Allgemeinen sollten der Theorie zufolge Übergeneralisierungen im Gebrauch des bestimmten Artikels wie in

(5) In meiner Freizeit jobbe ich in einem Studiumzentrum, wo ich den Kindern und den Jugendlichen beim Hausaufgaben helfe. ${ }^{16}$

häufig vorkommen. In der Theorie wird aber auch präzisiert, dass es sich dabei um „Überlegungen zum Transferpotential“ handelt, „,ie sich aus den theoretischen Überlegungen zum

(cf. Barton 2016: 175). Die französischen Probanden ersetzen den unbestimmten Artikel und den Nullartikel systematisch durch den bestimmten Artikel (cf. ibd.: 176).

${ }^{16}$ Dieses Beispiel stammt aus der Hausarbeit einer italienischen Masterstudentin (Niveau C1 des Gemeinsamen Europäischen Referenzrahmens (GER)). 
Nullartikel [...] schlussfolgern ließen“ (Stollhans 2012: 622). Empirische Untersuchungen werden also dringend benötigt.

Der theoretische Vergleich der Sprachsysteme lässt ferner die Vermutung zu,

3) dass Lerner mit Italienisch und Französisch als L1 nicht nur Probleme bei der Entscheidung für oder gegen einen Artikel (syntaktische Ebene), sondern auch bei der Wahl des passenden Artikels (semantische Ebene) in ihrer deutschen L2-Produktion haben können (cf. Barton 2016: 93).

\subsection{Design der Studie und Vorgehensweise}

Die vorliegende explorative Studie besteht aus drei Korpusanalysen, die darauf abzielen, den konkreten Gebrauch der deutschen Artikel seitens italienischer und französischer fortgeschrittener Lerner zu ermitteln, wobei sich das Augenmerk auf übergeneralisierte (d. h. von den korrigierenden bzw. annotierenden muttersprachlichen Linguisten/Experten gestrichene) Artikelformen, auf fehlende (d. h. hinzugefügte) explizite Artikelformen (= Übergeneralisierung des Nullartikels) und auf die Art der Artikel (korrigierte Form: bestimmt vs. unbestimmt oder umgekehrt) richtet. ${ }^{17}$

Die Untersuchung stützt sich auf drei Korpora: ${ }^{18}$

- $\quad$ FalkoEssayL2 + FalkoEssayL2WHIG (Humboldt-Universität zu Berlin);

- $\quad$ KoLas (Universität Hamburg);

- Prüfungsaufsätze aus dem laurea-specialistica-Studiengang (Universität Bergamo).

Die Falko-Korpora sind online abrufbar und können automatisch durchgesucht werden. Das KoLas-Korpus ist nach dem Anmeldungsverfahren online zugänglich, muss aber manuell ausgewertet werden. Das dritte habe ich zusammengestellt und manuell analysiert.

Obwohl es sich um eine korpuslinguistische Analyse handelt, besteht kein Anspruch auf eine quantitative Aussagekraft der Ergebnisse. Die Zahlen dienen dazu, Gebrauchstendenzen aufzuzeigen. Die Ergebnisse werden qualitativ ausgewertet.

Bei der Auswertung werden die vom Verfasser gemeinte Funktion und Struktur berücksichtigt, selbst wenn sie hin zur zielsprachlichen Version geändert werden. ${ }^{19}$ Bezüglich der Arti-

\footnotetext{
${ }^{17}$ Für Angaben zum genauen methodischen Verfahren cf. § 3.2.

${ }^{18}$ Für ihre tatkräftige Unterstützung bei dem Anmeldungsverfahren und bei Fragen zur Korpus-Recherche bedanke ich mich herzlich bei den Mitarbeitern des Zentrums für Sprachkorpora der Universität Hamburg, insbesondere bei Hanna Hedeland, sowie bei Dagmar Knorr, Besitzerin des KoLas-Korpus, und bei Martin Klotz, der für die Moodle- und Homepage-Verwaltung am Institut für deutsche Sprache und Linguistik der HumboldtUniversität zu Berlin zuständig ist.

${ }^{19}$ Hier ein Beispiel dafür (FalkoEssayL2WHIG, FRA INS, also ein Beispiel für die Hinzufügung eines fehlenden Artikels in einem FalkoEssayL2WHIG-Text eines französischen Verfassers):

tok und auf eine Seite können sie denken, dass man schwach und kein wirklicher Mensch ist, wenn sie Kriminalität als eine gute Sache sehen.

ZH1 und auf einer Seite können sie denken, dass man schwach und kein wirklicher Mensch ist, wenn sie in der Kriminalität eine gute Sache sehen.

tok gibt den Eintrag in der Originalversion wieder; ZH1 zeigt die erste Zielhypothese. Hinzugefügt wird hier in der, kategorisiert wird die fehlende Artikelform allerdings als „BESTIMMT SG - DIR OBJEKT POS“, denn sie betrifft die Singular-Ergänzung des direkten Objekts.
} 
kel-Hinzufügungen werden die Einträge aussortiert und nicht berücksichtigt, in denen der Artikel aufgrund syntaktischer Umformulierung des Satzes oder aufgrund der Aufsplitterung von falsch zusammengesetzten Formen hinzugefügt wird, weil die Ursprungsversion aufgrund anderer Abweichungen (nicht im Artikelgebrauch) geändert wird. ${ }^{20}$ Von den verändernden Korrekturen bestehender Artikel werden zur Analyse nur diejenigen in Betracht gezogen, welche die Veränderung der Art des Artikels (d. h. bestimmt vs. unbestimmt oder umgekehrt) veranlassen.

Im Falle der Falko-Korpora werden beide Zielhypothesen (cf. hierzu § 3.3.1) ausgewertet, da verschiedene Aspekte des Artikelgebrauchs in beiden korrigiert werden. In der ersten (ZH1) werden fehlende explizite Artikelformen hinzugefügt, allerdings wird die fehlende bzw. falsche Markierung der kontextabhängigen Definitheit erst in der zweiten (ZH2) korrigiert (cf. Reznicek et al. 2012: 46-47, 53); durch ZH2 werden alle evtl. gelöschten, ergänzten und veränderten Artikel, auch im Bezug auf Kollokationen (cf. Reznicek et al. 2012: 56) und auf Veränderungen in der Informationsstruktur (cf. ibd.: 59), ersichtlich.

\subsection{Textgrundlage}

Insgesamt werden 68 Texte italophoner und 23 Texte frankophoner Lerner untersucht.

\begin{tabular}{|l|l|l|}
\hline Korpus & Texte italienischer Lerner & Texte französischer Lerner \\
\hline FalkoEssayL2 & 4 & 17 \\
\hline FalkoEssayL2WHIG & 1 & 2 \\
\hline KoLas & 5 & 4 \\
\hline Prüfungsaufsätze & 58 & $/$ \\
\hline
\end{tabular}

Tabelle 2: Textgrundlage

\subsubsection{Falko-Korpora (Version 2.4) ${ }^{22}$}

Beide Falko-Lerner-Korpora werden in Betracht gezogen; in die Analyse werden insgesamt 24 Texte einbezogen, 19 von französischen und 5 von italienischen Verfassern.

Sowohl das FalkoEssayL2- als auch das FalkoEssayL2WHIG-Korpus bestehen aus argumentativen Aufsätzen von fortgeschrittenen Lernern des Deutschen. Die Erhebungen (für die hier relevanten Texte zwischen dem 27.07.2006 und dem 06.08.2008) erfolgten zum Teil während Feriensprachkurse an der Freien Universität Berlin und der Humboldt-Universität zu Berlin und zum Teil an ausländischen Universitäten und Goethe-Instituten (cf. Reznicek et al. 2012: 23).

Die durchschnittliche Sprachkompetenz ist hoch: 142 Verfasser der Texte von FalkoEssayL2 liegen im C1/C2-Bereich des Gemeinsamen Europäischen Referenzrahmens (GER), 105 im B2-Bereich (die Angaben eines Verfassers fehlen); 105 Verfasser der Texte von FalkoEssayL2WHIG weisen das Niveau B2 und 91 das Niveau C1/C2 auf. Das Niveau wird anhand

\footnotetext{
${ }^{20}$ Zum Beispiel wird die Formulierung „um ihre Tasche zu holen“ in der ZH2 zu „um ihr die Tasche zu klauen“ (aus einem FalkoEssayL2WHIG-Text eines italienischen Verfassers).

${ }^{21}$ In den nächsten Tabellen gilt Folgendes: „Texte italienischer Lerner“ = „Italienisch“; „Texte französischer Lerner“ = „Französisch“.

${ }^{22}$ Für eine ausführliche Darstellung cf. das online abrufbare Falko-Handbuch (cf. Reznicek et al 2012).
} 
eines Tests ermittelt, der vom Sprachenzentrum der Humboldt-Universität zu Berlin entwickelt wurde.

Jeder Text beinhaltet durchschnittlich 495,08 Tokens.

Zwischen den zwei Lerner-Korpora bestehen keine Unterschiede außer der Tatsache, dass im Falle von FalkoEssayL2WHIG das komplette Spektrum von Metadaten erhoben wurde und dass die Erhebungsorte mehrere sind.

Die Aufsätze betreffen vier, nicht zuvor angekündigte Themen, unter denen die Verfasser eines wählen; hier die Titel (cf. Reznicek et al. 2012: 24):

- Der Feminismus hat den Frauen mehr geschadet als genutzt.

- Kriminalität zahlt sich nicht aus.

- Die meisten Universitätsabschlüsse bereiten die Studenten nicht auf die wirkliche Welt vor. Sie sind deswegen von geringem Wert.

- Die finanzielle Entlohnung eines Menschen sollte dem Beitrag entsprechen, den er/sie für die Gesellschaft geleistet hat.

Den Verfassern stehen keine Hilfsmittel zur Verfügung, der verwendete Texteditor beinhaltet keine Rechtschreibkorrektur, und die Gesamtzeit der Prüfung beträgt 90 Minuten.

Die Falko-Korpora sind fehlerannotiert. Das Profil der Annotatoren wird nicht beschrieben. Allerdings ist zu entnehmen, dass die Annotationen z. B. im Falle eines anderen FalkoKorpus (Zusammenfassungen) im Rahmen von Magisterarbeiten erfolgen (cf. Reznicek et al. 2012: 19). Die Korrektur der Falko-Texte im Rahmen des Annotationsverfahrens ist sehr genau. Es werden zwei Zielhypothesen vorgeschlagen, wobei Zielhypothese „eine Interpretation der Lerneräußerung durch einen geschulten Annotator darstellt" (Reznicek et al. 2012: 38). ${ }^{23}$ Zuerst wird eine minimale Zielhypothese aufgestellt (ZH1), welche die Satzebene, die Orthographie und die Morphosyntax in Betracht zieht. Danach wird eine zweite Zielhypothese (ZH2) vorgeschlagen, die Textebene, Semantik, Pragmatik, Referenz, informationsstrukturelle Gliederung und Stil in der Originalversion berücksichtigt; diese soll sich den Äußerungen eines deutschen L1-Sprechers so weit wie möglich nähern (cf. Reznicek et al. 2012: 51). ${ }^{24}$

\subsubsection{KoLas-Korpus (Version 2.0)}

Das KoLas-Korpus liegt in der endgültigen Version 2.0 vor, die am 22.02.2017 online veröffentlicht wurde. Es besteht aus 854, auf Deutsch verfassten akademischen Texten, die von deutschen Schreibberatern kommentiert wurden. Diese Texte stammen aus 122, meist ausländischen Verfassern, die sich in dem Zeitraum September 2011-Dezember 2016 als Ratsuchende an die „Schreibwerkstatt Mehrsprachigkeit“ des Instituts für Interkulturelle Bildung der Fakultät für Erziehungswissenschaft an der Universität Hamburg gewendet haben. Die Ratsuchenden legen Texte vor, die sie für eine Prüfungsleistung benötigen

\footnotetext{
${ }^{23}$ Durch den Begriff „Zielhypothese“ wollen die Annotatoren des Korpus betonen, dass es sich dabei um eine der möglichen Varianten handelt, die vorgeschlagen werden können. Es wird somit die Subjektivität der Korrektur explizit thematisiert.

${ }^{24}$ Die komplexen Verben werden schließlich gesondert annotiert (ZHverb).
} 
(cf. Andresen/Knorr 2017: 2). ${ }^{25}$ Dieses Korpus hat als Sammlung von authentischen akademischen Texten in deutscher Sprache einen besonders hohen Stellenwert, denn die Texte können in vielen Fällen auch longitudinal untersucht werden (d. h.: Es liegen mehrere Texte vom selben Verfasser vor, oft nach mehreren Revisionsphasen). Alle Texte wurden anonymisiert, sind aber mit Metadaten wie Geschlecht, Abschluss, ausführlicher Sprachbiographie, Land der Hochschulreife, eventuellem Migrationshintergrund, Studienfach und Fachsemester verknüpft. ${ }^{26}$

Die für die vorliegende Studie in Frage kommenden Texte sind insgesamt 9, von 3 Verfassern (einer Studentin mit Französisch als L1 und zwei mit Italienisch als L1).27

\subsubsection{Prüfungsaufsätze}

Das Korpus der Prüfungsaufsätze der Universität Bergamo besteht aus insgesamt 58 Arbeiten zum gelenkten Schreiben (ca. 1,5 Seiten jeweils, handgeschrieben und ohne Hilfsmittel verfasst) von Studierenden aus dem laurea-specialistica-Studiengang der Fakultät für Fremdsprachen und Literaturen, 33 aus dem ersten Jahr (Prüfungstermine: September 2010 und Mai 2015) und 25 aus dem zweiten Jahr (Prüfungstermine: September 2010, Mai 2014, September 2014 und Mai 2015). ${ }^{28}$ Diese Rahmenbedingungen setzen ein theoretisches Sprachniveau von ca. C1 oder sogar C2 des Gemeinsamen Europäischen Referenzrahmens (GER) voraus. Die Anleitungen sind unterschiedlich: Im ersten Jahr müssen Hörtexte zusammengefasst werden. Die Rahmenthemen sind: die Rolle des Goethe-Instituts innerhalb und außerhalb Deutschlands (Studiengang LLEP, Prüfungstermin September 2010; 3 Klausuren); die RomaMinderheit in Europa (Studiengang Fremdsprachen, Prüfungstermin September 2010; 5 Klausuren); Seminare für Migranten (Prüfungstermin Mai 2015; 25 Klausuren). Im zweiten Jahr müssen ein Hörtext und ein Lesetext zu einem gleichen Rahmenthema zusammengefasst werden. Die Rahmenthemen sind: die Team-Arbeit (Prüfungstermin September 2014; 2 Klausuren); der Kontakt zu der Natur, um vom Stress abzuschalten (Prüfungstermin Mai 2014; 7 Klausuren); Noten und Zeugnisse an Schulen (Prüfungstermin September 2010; 2 Klausuren). Beim Prüfungstermin vom Mai 2015 (14 Klausuren) besteht die Aufgabe darin, eine EUGraphik über Umweltprobleme und Plasticktüten zu beschreiben und zu kommentieren.

Alle Klausuren wurden von Deutsch-Lektorinnen korrigiert und bewertet.

\footnotetext{
25 Dadurch, dass es sich um Studierende an einer deutschen Universität handelt, geht man von dem Mindestniveau von B2 des Gemeinsamen Europäischen Referenzrahmens (GER) (DSH 2) aus.

${ }^{26}$ Weitere Informationen zu diesem Korpus finden sich in Andresen/Knorr 2017.

27 Die französischsprachige Studentin (im 10. Fachsemester) kommt aus der Republik Guinea, wo sie ihre Hochschulreife erworben hat, und ihre 4 Texte sind Teile ihrer BA-Abschlussarbeit in der Sozialökonomie zum Thema „Entwicklungshilfe in Guinea“ (jeweils 3, 3, 3 und 4 Seiten).

Die erste italienische Studentin (im 6. Fachsemester) hat in Italien auf Bachelor und Master studiert und kam nach 6 Monaten Deutschkurs zur Promotion nach Deutschland. Der „Schreibwerkstatt Mehrsprachigkeit“ legt sie das erste Kapitel ihrer Dissertation vor (Titel: „Das Leben von Zomino da Pistoia“; insgesamt 14 Textseiten) sowie die Überarbeitung der ersten 3 Textseiten.

Die zweite italienische Studentin (im 9. Fachsemester im Studiengang Archäologie) hat ebenfalls in Italien ihre Hochschulreife erworben. Sie reicht der Schreibwerkstatt drei Texte zur Korrektur ein: die ausformulierte Version eines Referats über Claudius (5 Textseiten), eine Hausarbeit über die deutsche Übersetzung eines Briefs von Plinius dem Jüngeren (5 Textseiten davon wurden kommentiert) und eine vollständige Hausarbeit über die Thermalbäder im alten Rom (7 Textseiten).

${ }^{28}$ Die Klausuren aus dem Jahr 2010 stammen offiziell aus dem 4. und 5. Jahr, da vor dem Bologna-Prozess die alte Prüfungsordnung (5 Jahre-Studium ohne Einteilung in laurea triennale und laurea specialistica) galt.
} 


\subsection{Ergebnisse}

Im Folgenden werden die Ergebnisse dargestellt; deren Auswertung und Diskussion erfolgen in $\S 3.5$.

\subsubsection{FalkoEssayL2}

Gegen die Hypothesen, die sich von der theoretischen Darstellung des kontrastiven Artikelgebrauchs ableiten lassen ( $\S 3.1$ ), wird im Laufe des Korrekturverfahrens keiner der von den italienischen und französischen Verfassern verwendeten Artikel hin zu einer theoretisch sprachgeformten Zielversion gelöscht.

Umgekehrt wird der Nullartikel 18 mal in Essays italienischer Lerner und 52 mal in Essays französischer Verfasser durch eine andere Artikelform ersetzt. In folgender Tabelle werden die einzelnen relevanten Artikel-Hinzufügungen (= Korrektur des Nullartikels) qualitativ kategorisiert, nämlich hinsichtlich der Art sowie der Funktion und Struktur der jeweils betroffenen NP.

\begin{tabular}{|l|l|l|}
\hline NP & Italienisch & Französisch \\
\hline BESTIMMT SG [Singular] - SUBJEKT POS [Position] & 8 & 12 \\
\hline BESTIMMT SG - SUBJEKT POS MIT ADJ [Adjektiv] & & 2 \\
\hline BESTIMMT PL [Plural] - SUBJEKT POS & 2 & 1 \\
\hline BESTIMMT SG - DIR [direktes] OBJEKT POS & & 3 \\
\hline BESTIMMT SG - DIR OBJEKT POS MIT ADJ & 1 & 1 \\
\hline $\begin{array}{l}\text { BESTIMMT SG - INDIR [indirektes] OBJEKT od. PRÄP } \\
\text { [PräpOsition] }\end{array}$ & 13 \\
\hline BESTIMMT SG - INDIR OBJEKT od. PRÄP POS MIT ADJ & 1 & 1 \\
\hline BESTIMMT PL - INDIR OBJEKT od. PRÄP POS MIT ADJ & & 2 \\
\hline UNBESTIMMT SG - SUBJEKT POS & 1 \\
\hline UNBESTIMMT SG - SUBJEKT POS MIT ADJ & 1 \\
\hline UNBESTIMMT SG - DIR OBJEKT POS & 1 & 1 \\
\hline UNBESTIMMT SG - DIR OBJEKT POS MIT ADJ & 1 \\
\hline $\begin{array}{l}\text { UNBESTIMMT SG - DIR OBJEKT POS MIT PARTIZIPI- } \\
\text { ALKONSTRUKTION }\end{array}$ & & 1 \\
\hline UNBESTIMMT SG - INDIR OBJEKT od. PRÄP POS & 1 \\
\hline UNBESTIMMT SG - INDIR OBJEKT od. PRÄP POS MIT & 1 & 1 \\
\hline
\end{tabular}

Tabelle 3: Eingefügte explizite Artikelformen anstelle vom Nullartikel (Korpus FalkoEssayL2)

Selbst wenn die Verteilung des zielsprachlich abweichenden Nullartikels ein breites Spektrum abdeckt und NPs mit mehreren Formen und Funktionen betrifft, besteht der überwiegende 
Teil der Abweichungen im Gebrauch des bestimmten Artikels. Die Artikel fehlen am häufigsten in minimalen Singular-Subjekt-NPs, die keine Adjektive, Partizipialkonstruktionen oder sonstige Attribute beinhalten. ${ }^{29}$ Hier ein typisches Beispiel:

(6) tok man kann deshalb nicht wissen in wie weit Feminismus die Rolle der Frauen geaendert hat.

ZH1 Man kann deshalb nicht wissen, inwieweit der Feminismus die Rolle der Frauen geändert hat. ${ }^{30}$

Dies legt nahe, dass das Vorhandensein eines Adjektivs in der NP nicht mit einer häufigeren Auslassung expliziter Artikelformen einhergeht.

Die französischen Verfasser haben genau so viele Schwierigkeiten auch bei Singular-NPs, welche die Funktion einer Präpositionalangabe oder eines indirekten Objektes erfüllen und den bestimmten Artikel benötigen, wie in (7)

(7) tok aber bereuen, dass die praxistischen Fächer nicht mehr Wert haben, da sie sind immer wichtig, für Alltagsleben, aber auch weil man Handarbeiter braucht.

ZH1 aber bereuen, dass die praktischen Fächer nicht mehr Wert haben, da sie immer wichtig für das Alltagsleben sind, aber auch, weil man Handarbeiter braucht.

Von den vielen Änderungen bestehender Artikel (ca. $11 \%$ : 28 der 275 Artikel in den Texten italienischer Verfasser und 121 der 1023 Artikel in den Texten französischer Verfasser) sind nach dem Sortieren-Verfahren (cf. § 3.2) nur 7 der insgesamt 149 Einträge (weniger als $5 \%$ ) näher zu betrachten, inklusive derjenigen, in denen anderes Sprachmaterial durch Artikel ersetzt wird (da auch dadurch Abweichungen im Artikelgebrauch ersichtlich werden):

(8) tok Man konnte dann fragen, ,wie kann man sein Leben mit Erfolg führen, wenn man sich die Kopf in sein eigenen Träume stecken lässt?“

ZH2 Man könnte dann fragen, wie man ein erfolgreiches Leben führen kann, wenn man mit dem Kopf in seinen eigenen Träumen steckt?

(9) tok oder diese doppelte Aktivität (Arbeit im Büro und Hausarbeit) vermeiden möchten?

ZH2 oder die doppelte Belastung (Arbeit im Büro und Hausarbeit) vermeiden möchten?

\footnotetext{
${ }^{29} \mathrm{Zu}$ den minimalen NPs gehören auch Ausdrücke, die aus einem Nomen und einem Determinativ bestehen (cf. Zifonun 1997: 1929).

${ }^{30} \mathrm{Im}$ Falle der Beispiele aus den Falko-Korpora wird sowohl die Originalversion (tok) als auch die korrigierte Version ( $\mathrm{ZH} 1$ oder $\mathrm{ZH}$ ) wiedergegeben, wobei die hinzugefügten Artikel unterstrichen werden und das durch Artikel ersetzte Sprachmaterial kursiv formatiert wird. Alle Originalversionen werden nicht verändert und weisen deshalb viele andere Fehler auf.
} 
(10) tok Wirtschaftswissenschaftler der liberalen Schule denken, dass der Markt am besten funktionnert, wenn er nach diesem Gesetz des Angebots und der Nachfrage geregelt wird.

ZH2 Wirtschaftswissenschaftler der liberalen Schule denken, dass der Markt am besten funktioniert, wenn er nach dem Gesetz des Angebots und der Nachfrage geregelt wird.

(11) tok Diese Femministinnen haben den Feminismus manchmal zu dieser Karikatur gebracht. Ein Slogan einer Bewegung in Frankreich heute.

ZH2 Diese Feministinnen haben den Feminismus manchmal zu dieser Karikatur gemacht. Der Slogan einer Bewegung in Frankreich heute.

(12) tok Selbst wenn man dieses Praktikum und diese Probezeit einstellt, werden wir das Problem wieder treffen.

ZH2 Selbst wenn man ein Praktikum und eine Probezeit einführt, wird man wieder auf dieses Problem treffen.

(13) tok Das Problem kommt, denke ich, von den humanistischen Wissenschaften.

ZH2 Ein Problem haben, denke ich, die Geisteswissenschaften.

(14) tok als den Künstler? Ist aber Kunst nicht genauso wichtig wie in einem sauberen Haus zu leben? Ist der Firmenleister nicht so wichtig wie der Handwerker, (sonst wer würde dann ihm seine Entlohnung geben?)

ZH2 als einem Künstler? Ist aber Kunst nicht genauso wichtig, wie in einem sauberen Haus zu leben? Ist ein Firmenchef nicht genauso wichtig wie ein Handwerker, (wer würde ihm denn sonst seine Entlohnung geben?)

Es ist festzuhalten, dass in einigen Fällen Demonstrativa und Possessiva durch (bestimmte und unbestimmte) Artikelformen ersetzt werden; allerdings ist einmal die Gegenkorrektur belegt (ein bestimmter Artikel wird durch ein Demonstrativum korrigiert). Nur fünfmal wird die (Un)bestimmtheit korrigiert, wobei es viermal um die Ersetzung des bestimmten Artikels durch den unbestimmten Artikel geht.

\subsubsection{FalkoEssayL2WHIG}

Auch in den 3 Texten italienischer und französischer Verfasser vom FalkoEssayL2WHIGKorpus werden keine Artikel gelöscht, so dass auch hier die Übergeneralisierung des Artikelgebrauchs ausgeschlossen werden kann.

In den zwei Texten französischer Verfasser sind 9 und in dem Text des italienischen Verfassers 4 Hinzufügungen (= Korrektur des übergeneralisierten Nullartikels) vorzufinden, welche die folgende Tabelle zusammenfasst. 


\begin{tabular}{|l|l|l|}
\hline NP & Italienisch & Französisch \\
\hline BESTIMMT SG - SUBJEKT POS & 2 & 4 \\
\hline BESTIMMT PL - SUBJEKT POS & 1 & \\
\hline BESTIMMT SG - DIR OBJEKT POS & 1 & 1 \\
\hline BESTIMMT SG - INDIR OBJEKT od. PRÄP & & 1 \\
\hline UNBESTIMMT SG - SUBJEKT POS & & 2 \\
\hline UNBESTIMMT SG - DIR OBJEKT POS & & 1 \\
\hline
\end{tabular}

Tabelle 4: Hinzugefügte explizite Artikelformen anstelle vom Nullartikel in den analysierten Texten aus dem FalkoEssayL2WHIG-Korpus

Auch diesmal überwiegt als Sprachkontext der übergeneralisierten Nullartikel-Okkurrenzen die minimale Singular-Subjekt-NP, wobei der zielsprachlich korrekte bestimmte Artikel etwas mehr betroffen ist.

Der Prozentsatz der geänderten Formen liegt bei ca. $22 \%$, 37 der insgesamt 167 Artikelvorkommnisse. Nur wenige dieser 37 Änderungen sind nicht durch Veränderungen im gesamten Sprachkontext bedingt bzw. nicht auf Kasus-, Genus- oder Numerus-Fehler zurückzuführen, wie der Auflistung aller Beispiele (inkl. derjenigen, in denen anderes Sprachmaterial als Artikel in der Ursprungsversion vorliegt) gleich zu entnehmen ist:

(15) tok kann man für, wie lang, dass sein Leben dauert, allein ins Gefängnis

ZH1 kann man so lang, wie das Leben dauert, allein ins Gefängnis kommen

(16) tok weil diese Jugend die Zukunft der Gesellschaft ist.

ZH2 weil die Jugend die Zukunft der Gesellschaft ist.

Es fällt auf, dass die Fehler auch nicht durch die falsche Zuweisung der (Un)bestimmtheit entstehen. Diese letzte Veränderung trifft nur auf 3 Einträge zu und erscheint erst auf der ZH2-Ebene und durch Veränderungen des Wortschatzes bzw. inhaltliche Ergänzungen:

(17) tok Der Staat musste den Familien in Schwerigkeit helfen, weil ein Vater oder eine Mutter die Pflicht, ihre kinder zur Schule zu schicken [...] hat.

ZH2 Der Staat muss den Familien in Schwierigkeiten helfen, weil die Eltern die Pflicht haben, ihre Kinder zur Schule zu schicken [...] haben.

(18) tok wer kann - oder besser - wer hat Zeit, ein bisschen sein Wissen mit einem Jungen, einem Mädchen zu verbringen?

ZH2 Wer kann oder besser wer hat also Zeit dafür, sein Wissen mit den Kindern zu teilen?

(19) tok wer will ein Freund sein, wer will nur kennen, dies Art von Mensch?

ZH2 wer will der Freund eines solchen Menschen sein und wer will nur die Sorte Menschen kennen? 


\subsubsection{KoLas-Korpus}

Folgende Tabelle zeigt die Funktion und die Art der NPs, in denen der Nullartikel in den berücksichtigten KoLas-Texten übergeneralisiert worden war und folglich explizite Artikelformen hinzugefügt wurden.

\begin{tabular}{|l|l|l|}
\hline NP & Italienisch & Französisch \\
\hline BESTIMMT SG - SUBJEKT POS & 3 & 2 \\
\hline BESTIMMT SG - SUBJEKT POS MIT ADJ & & 3 \\
\hline BESTIMMT SG - DIR OBJEKT POS & 1 & 2 \\
\hline BESTIMMT SG - DIR OBJEKT POS MIT ADJ & & 1 \\
\hline BESTIMMT SG - INDIR OBJEKT od. PRÄP & 2 & 1 \\
\hline $\begin{array}{l}\text { BESTIMMT SG - INDIR OBJEKT od. PRÄP POS MIT } \\
\text { ADJ }\end{array}$ & 3 & \\
\hline UNBESTIMMT SG - SUBJEKT POS MIT ADJ & 1 & 1 \\
\hline UNBESTIMMT SG - DIR OBJEKT POS MIT ADJ & 1 & \\
\hline UNBESTIMMT SG - INDIR OBJEKT od. PRÄP POS & 1 & \\
\hline $\begin{array}{l}\text { UNBESTIMMT SG - INDIR OBJEKT od. PRÄP POS } \\
\text { MIT ADJ }\end{array}$ & 2 & \\
\hline
\end{tabular}

Tabelle 5: Hinzugefügte explizite Artikelformen (anstelle von dem übergeneralisierten Nullartikel) in den KoLas-Korpus-Texten italienischer und französischer Verfasser

Die Studentin mit Französisch als L1 hat deutlich Probleme mit der Verwendung des bestimmten Artikels (alle Einträge betreffen diese Artikelform), insbesondere in Subjekt-NPs. Die Beispiele sind ähnlich wie (6):

(20) Deshalb hat der guineische Wirtschaftsplan für die ökonomische Entwicklung des Landes bis heute nachhaltig beeinflusst. ${ }^{31}$

Solche Abweichungen kommen auch diesmal auch in den Texten italienischer Verfasser vor, wobei 2 der insgesamt 3 Einträge eine Auflistung betreffen:

(21) Der Zweck dieser Hausarbeit ist das Verständnis, die Analyse und die Kontextualisierung eines Briefes des Plinius der Jüngere.

Nur das erste Element wird mit dem Artikel versehen, obwohl die folgenden einen anderen Artikel verlangen.

Die Arbeiten der italienischen Studentinnen weisen Fehler sowohl bzgl. der bestimmten als auch der unbestimmten Artikelformen auf. Schließlich ist anzumerken, dass 2 der 3 fehlenden bestimmten Artikel in Präpositionalphrasen mit der Struktur im...Jh. in Verbindung stehen;

\footnotetext{
${ }^{31}$ Die Beispiele aus diesem Korpus werden gleich in der korrigierten Form wiedergegeben. Die hinzugefügten Artikel werden unterstrichen.
} 
der Nullartikel in dieser Konstruktion ist weder in der Ausgangs- noch in der Zielsprache zulässig und deutet deshalb auf die Übergeneralisierung des Nullartikels hin.

Während insgesamt 23 explizite Artikelformen hinzugefügt werden, werden nur 2 gelöscht, und sogar in besonderen Kontexten: in einem Titel (Beispiel (22)) und in einer Kollokation (Beispiel (23)):

(22) Die Jugendzeit und Bildung

(23) um die Hilfe zu bekommen

In den Texten der italienischen Studentinnen wird fünfmal der bestimmte Artikel durch Possessiva ersetzt (geänderte Artikel); es handelt sich um einen der wesentlichen Unterschiede im Artikelgebrauch zwischen Deutsch und Italienisch. Hier die Einträge:

(24) zusammen mit dem [seinem] Bruder

(25) um sich erfolgreich der [seinem] humanistischen Tätigkeit zu widmen

(26) in der Werkstatt des [seines] Freundes

(27) nach der [seiner] Rückkehr nach Florenz

(28) bei dem [ihrem] Begräbnis

Hinzu kommen zwei Okkurrenzen der Veränderung der (Un)bestimmtheit:

(29) Er war ein [der] unehelicher Sohn des Notars

(30) Wenn man versucht, die [eine] Schlussfolge zu erreichen

$\mathrm{Zu}$ zitieren ist der Kommentar des Schreibberaters bzgl. Beispiel (30): „Du willst hier eine allgemeine Aussage machen, daher würde ich den unbestimmten Artikel benutzen“. Allerdings handelt es sich um das einzige Beispiel, bei dem eine allgemeine Aussage mit dem bestimmten Artikel eingeleitet wird.

\subsubsection{Prüfungsaufsätze}

Die 7 Artikelformen, die in den untersuchten Prüfungsaufsätzen geändert werden, betreffen die Art des Artikels (+/- Bestimmtheit) nicht. Es handelt sich um Verwechselungen von Artikeln/Pronomen (ein vs. eines/einer), Relativpronomen/Konnektoren (die vs. dass) und Formen der Wiederaufnahmen (das vs. was), die auf die Übernahme italienischer Strukturen und auf andere Interferenzen aus dem italienischen Sprachsystem zurückzuführen sind. Nur einmal wird die durch eine ersetzt, nämlich in der Subjekt-NP „Eine [Die] 2010 von der APA/EU-Kommission veröffentlichte Graphik“; die gleiche NP in anderen Aufsätzen, selbst bei der Ersterwähnung, wird allerdings nicht korrigiert, denn es liegt ja eine Graphik vor und es ist durchaus möglich, dass sie als Bestandteil des Textes (= identifizierbarer Referent) aufgefasst wird. 
In diesem Korpus finden sich zum ersten Mal einige Einträge, in denen vorhandene explizite Artikelformen gelöscht werden: ${ }^{32}$

\begin{tabular}{|c|c|c|}
\hline NP & ITA LM I/IV Jahr & ITA LM II/V Jahr \\
\hline $\begin{array}{l}\text { BESTIMMT SG - SUB- } \\
\text { JEKT POS }\end{array}$ & $3 *$ & $5^{*}$ \\
\hline $\begin{array}{l}\text { BESTIMMT PL - SUB- } \\
\text { JEKT POS MIT ADJ }\end{array}$ & $\begin{array}{l}1 \text { (nicht alle die Berliner } \\
\text { Einwohner) }\end{array}$ & \\
\hline $\begin{array}{l}\text { BESTIMMT SG }- \text { DIR } \\
\text { OBJEKT POS }\end{array}$ & & 1 (hörte sie das Musik) \\
\hline $\begin{array}{l}\text { BESTIMMT SG - INDIR } \\
\text { OBJEKT od. PRÄP }\end{array}$ & $3 *$ & $3 *$ \\
\hline $\begin{array}{l}\text { BESTIMMT SG - INDIR } \\
\text { OBJEKT od. PRÄP POS } \\
\text { MIT ADJ }\end{array}$ & $\begin{array}{l}1 \text { (Plural-NP: Im anderen } \text { Ländern })^{33}\end{array}$ & \\
\hline $\begin{array}{l}\text { BESTIMMT PL - INDIR } \\
\text { OBJEKT od. PRÄP }\end{array}$ & $\begin{array}{l}1 \text { (für die deutschen Stipen- } \\
\text { diaten) } \\
1 \text { (Plural-NP: Im Staaten } \\
\text { wie) }\end{array}$ & \\
\hline $\begin{array}{l}\text { UNBESTIMMT SG - DIR } \\
\text { OBJEKT POS }\end{array}$ & & 1 (ein Sport treiben) \\
\hline $\begin{array}{l}\text { UNBESTIMMT SG - DIR } \\
\text { OBJEKT POS MIT ADJ }\end{array}$ & & $\begin{array}{l}1 \text { (Plural-NP: um eine gute } \\
\text { Synergie-Effekte } \mathrm{zu} \text { errei- } \\
\text { chen) }\end{array}$ \\
\hline $\begin{array}{l}\text { UNBESTIMMT SG - IN- } \\
\text { DIR OBJEKT od. PRÄP } \\
\text { POS }\end{array}$ & $\begin{array}{l}1 \text { (in einen Kontakt zu kom- } \\
\text { men) }\end{array}$ & \\
\hline
\end{tabular}

Tabelle 6: Gelöschte Artikel in den Prüfungsaufsätzen

Kursiv markiert werden die Einträge, die aus nicht ausreichenden Klausuren stammen. Das Gesamtniveau der Verfasser dieser Texte liegt also weit unter den Erwartungen und könnte diese Abweichungen in der Lernervarietät erklären. Die mit einem Sternchen versehenen Einträge (14 von 22, alle den bestimmten Singularartikel betreffend) beziehen sich auf Artikel bei Ortsbezeichnungen (meist Stadt- und Staatsnamen). Nur zum Teil lassen sich diese Abwei-

\footnotetext{
${ }^{32}$ Die Vorkommnisse des Ausdrucks im Jahr werden nicht berücksichtigt, da die nicht wohlgeformte, häufig vorkommende Form im [z. B. 2010] zweideutig interpretiert werden kann: als Fehler beim Artikelgebrauch oder als Auslassung des Substantivs Jahr. Diese Schwierigkeit ist deutlich auf das italienische Sprachsystem zurückzuführen, das mit und ohne Jahr den Artikel verlangt.

${ }^{33}$ Diese Beispiele sind aus der Auswertung auszuschließen. Möglicherweise handelt es sich bei den ersten zwei um eine unklar geschriebene Form von in. Die Plural-NP mit eine kam vermutlich durch eine nicht korrigierte Veränderung des Inhaltes zu Stande.
} 
chungen durch italienische Interferenzen erklären (cf. *im Europa/Heidelberg vs. it. in Europa/a Heidelberg). ${ }^{34}$

In fünf Fällen wird der (bestimmte und unbestimmte) Artikel doch übergeneralisiert: „für die deutschen Stipendiaten“, „nicht alle die Berliner Einwohner“ (was regional eventuell akzeptabel ist), „,hörte sie das Musik“, „ein Sport treiben“ und „in einen Kontakt zu kommen“. Die letzten drei Einträge sind Kollokationen, die interessanterweise nicht nur im Deutschen, sondern auch im Italienischen ohne Artikel vorkommen. Nur beim ersten Beispiel geht es um einen generischen Ausdruck im Plural (was aus dem Kontext hervorgeht), die fälschlicherweise mit dem bestimmten Artikel und nicht mit dem Nullartikel eingeleitet wird.

Auch in diesem Korpus überwiegen die Einträge der hinzugefügten expliziten Artikelformen (= Korrektur des übergeneralisierten Nullartikels), die in der folgenden Tabelle zusammengefasst werden.

\begin{tabular}{|l|l|l|}
\hline NP & ITA LM I/IV Jahr & ITA LM II/V Jahr \\
\hline BESTIMMT SG - SUBJEKT POS & 7 & 2 \\
\hline BESTIMMT SG - SUBJEKT POS MIT ADJ & 1 & \\
\hline BESTIMMT SG - DIR OBJEKT POS & 2 & 2 \\
\hline BESTIMMT PL - DIR OBJEKT POS & 1 \\
\hline $\begin{array}{l}\text { BESTIMMT SG - DIR OBJEKT POS MIT } \\
\text { ADJ }\end{array}$ & 1 & 1 \\
\hline BESTIMMT SG - INDIR OBJEKT od. PRÄP & 11 & 7 \\
\hline $\begin{array}{l}\text { BESTIMMT SG - INDIR OBJEKT od. PRÄP } \\
\text { POS MIT ADJ }\end{array}$ & 1 \\
\hline $\begin{array}{l}\text { BESTIMMT PL - INDIR OBJEKT od. PRÄP } \\
\text { UNBESTIMMT SG - DIR OBJEKT POS MIT } \\
\text { ADJ }\end{array}$ & 1 & 2 \\
\hline $\begin{array}{l}\text { UNBESTIMMT SG - INDIR OBJEKT od. } \\
\text { PRÄP MIT ADJ }\end{array}$ & 1 \\
\hline
\end{tabular}

Tabelle 7: Hinzugefügte explizite Artikelformen (anstelle vom Nullartikel) in den Prüfungsaufsätzen

Ähnlich wie in den anderen Korpora fehlen auch hier viele explizite Artikelformen (9 von 40) in der Subjekt-Position bei minimalen NPs. Die Schwierigkeiten betreffen, wie auch an den gelöschten Artikeln deutlich wird, Eigennamen (und keine generischen Ausdrücke). ${ }^{35}$ In diesem Korpus fehlen auch viele bestimmte Artikel in Präpositionalphrasen, die mit dem Nullar-

\footnotetext{
34 Witwicka-Iwanowska (2012: 14) stellt im Falle von polnischen Lernern ebenfalls Fehler und Fehlertypen fest, die nicht durch Interferenz erklärt werden können.

35 Genauer: den Namen einer Hochschule, die Pluralbezeichnungen [die] Österreicher und [die] Niederlande sowie [die] EU. Gerade die häufige Erwähnung der EU/EU-Kommission (als Quelle der Graphik, welche die Grundlage von 14 Prüfungsaufsätzen ist) führt zur überdurchschnittlichen Zahl der Abweichungen in präpositionalen NPs: 9 der insgesamt 18 Einträge betreffen die Ausdrücke aus/von/in/zu [der] EU und von/in [der] EUKommission.
} 
tikel erscheinen; problematisch scheint die Verbindung mit EU/EU-Kommission zu sein. ${ }^{36}$ Zwei Einträge betreffen eine Kollokation ( $z u[\mathrm{r}]$ Schule gehen) und den Artikelgebrauch mit den Monaten (im August); die Fehlerquelle ist dabei die jeweilige italienische Entsprechung. Die Abweichungen in den anderen Kategorien sind im Vergleich zu den besprochenen Präpositionalphrasen und Subjekt-NPs deutlich marginal.

\subsection{Auswertung der Ergebnisse}

Bevor nun die in $\S 3.1$ formulierten Hypothesen wieder aufgegriffen werden, ist daran zu erinnern, dass die zu Grunde gelegte empirische Basis keine Verallgemeinerungen rechtfertigt. Vielmehr handelt es sich um eine explorative Studie, aus der sich erste Anhaltspunkte für mögliche Tendenzen im deutschen L2-Artikelgebrauch durch Lerner mit Italienisch und Französisch als L1 ergeben. Vorab ist ebenfalls festzuhalten, dass typische zielsprachliche Abweichungen im Artikelgebrauch (s. unten) nur teilweise auf den Artikelgebrauch in der L1 (negative Interferenz) zurückzuführen sind. Solche Fremdheitsmerkmale (für diesen Begriff cf. Ehlich u. a. 2007 [1986]) charakterisieren v. a. die erste Phase des Spracherwerbs (Imitation/Transposition; cf. Steinhoff 2003). ${ }^{37}$ Dies erklärt, warum sie besonders in nicht ausreichenden Klausuren (des Korpus von Prüfungsaufsätzen) vorkommen. Das im Allgemeinen hohe Niveau der hier berücksichtigten L2-Lerner geht also mit einer niedrigen Anzahl an negativen Interferenzen einher.

Alle drei, von der Theorie abgeleiteten Annahmen finden in den analysierten Lernertexten keine Bestätigung.

- Der Gebrauch des deutschen bestimmten Artikels in den untersuchten akademischen Texten von fortgeschrittenen Lernern mit Italienisch und Französisch als L1 ist weder überrepräsentiert noch übergeneralisiert (gegen Hypothese 2). ${ }^{38}$ Die Beispiele der gelöschten Artikelformen betreffen Sonderkontexte: Staatsnamen (Interferenz aus dem Italienischen) und Kollokationen (die nicht als feste Strukturen erkannt werden). Nur Besitz-Referenzen (die 5 Einträge der KoLas-Texte der italienischen Studentinnen) werden durch den bestimmten Artikel anstatt durch die Possessiva eingeleitet. ${ }^{39}$

- Generische Ausdrücke sind selten die Kontexte, in denen Abweichungen im konkreten L2-Artikelgebrauch vorkommen (gegen Hypothese 1).

- $\quad$ Nur sporadisch lassen sich Schwierigkeiten bei der Wahl der bestimmten vs. unbestimmten Artikelform feststellen (gegen Hypothese 3). Die Fälle in den Prüfungsaufsätzen sind sehr speziell, die 2 Beispiele aus den KoLas-Texten belegen beide Korrektur-Richtungen. Die 3 Veränderungen der (Un)bestimmtheit in den Texten des FalkoEssayL2WHIGKorpus ergeben sich aus inhaltlichen und lexikalischen Veränderungen. Nur in den Texten des FalkoEssayL2-Korpus kann man in 4 der 5 Fälle der (Un)bestimmtheit-Korrektur

\footnotetext{
${ }^{36}$ Auch in dem KoLas-Korpus-Text der französischsprachigen Studentin kommt EU-Kommission in einer Subjekt-NP mit dem Nullartikel vor.

37 Steinhoff untersucht allerdings den Erwerb der Wissenschaftssprache.

38 Witwicka-Iwanowska (2012: 16) stellt ebenfalls fest, dass einige Fehler und Fehlertypen weder durch Interferenz noch durch Übergeneralisierung zu erklären sind.

${ }^{39}$ Hingegen verwenden Lerner mit Französisch als L1 Possessiva und Demonstrativa z. T. zu häufig (cf. auch Barton 2016: 176), aber auch diesbezüglich sind die Zahlen sehr niedrig.
} 
von einer Übergeneralisierung des bestimmten Artikels auch zur Enkodierung von generischen Referenten ausgehen.

Zur Interpretation der Tatsache, dass der Artikelgebrauch nicht überrepräsentiert ist bzw. übergeneralisiert wird, bieten sich mindestens zwei Optionen an.

- Zunächst stellt sich die Frage, ob mit einer steigenden Akzeptanz des bestimmten Artikels auch bei generischen Ausdrücken im Deutschen zu rechnen ist; dagegen scheinen aber die Ergebnisse von Barton 2016 zu sprechen, denen zufolge konservativere deutsche L1-Sprecher den bestimmten Artikel häufiger akzeptieren; auch Barton et al. (2015: 165166) stellen die Tendenz fest, dass eher ältere und weniger ausgebildete Probanden den bestimmten Artikel häufiger dulden.

- Eine andere Option betrifft die Semantik der Versionen mit dem bestimmten Artikel und mit dem Nullartikel. In der Literatur wird z. T. angenommen, dass sie semantisch gleich sein können: Im Fall von zählbaren Pluralnomen und Massennomen ergebe die Wahl des bestimmten Artikels oder des Nullartikels keinen semantischen Unterschied, wie jeweils in (31) und (32):

(31) Die Biber bauen Dämme. / Biber bauen Dämme.

(32) Die Milch ist weiß. / Milch ist weiß. (cf. Longobardi 1994: 653)

Beispiel (32) wird allerdings von anderen Linguisten als fraglich angesehen (cf. Oosterhof 2004: 2).

Das zweite Ergebnis ist, dass in allen Korpora zielsprachlich explizite Artikelformen fehlen, wobei der Nullartikel übergeneralisiert wird. Am meisten sind minimale Singular-NPs in Subjekt-Position betroffen; hinzu kommen (nicht als solche erkannte) Kollokationen, Auflistungen und NPs mit Eigennamen.

Auch diesbezüglich bieten sich unterschiedliche Erklärungsmöglichkeiten.

- Die hier berücksichtigten Lerner sind im Erwerbsprozess weit genug fortgeschritten, um zu bemerken, dass im L1-Deutsch manchmal ein Nullartikel steht, wo sie aufgrund ihrer eigenen L1 eine explizite Artikelform erwarten würden, und bemühen sich in ihrer L2Produktion um eine entsprechend erhöhte Verwendung des Nullartikels im Deutschen, wobei sie diese teils übergeneralisieren.

- Durch die Zielsprache bedingte Übergeneralisierungen kommen gerade bei rein sprachlichen Elementen vor (cf. Roncoroni 2011: 85). In diesem Zusammenhang könnte man mit Ehlich (2007 [1986]) von Xenismen sprechen, d. h. Fremdheitsmerkmalen, die lediglich durch die Zielsprache zu Stande kommen ${ }^{40}$ und selbst fortgeschrittene Fremdsprachenlerner auf den höchsten Erwerbstufen noch als Nicht-Mitglieder der Sprachgemeinschaft der jeweiligen Zielsprache erkennen lassen (cf. Roncoroni 2010: 183, 196).

- Allerdings ist es durchaus möglich, dass sich Artikeleinsparungen im heutigen L1Deutsch etablieren: Dürscheid (2003) belegt, wie verbreitet die Tendenz dazu ist. Beispielsweise in SMS sind Artikelauslassungen nicht nur auf Sprachökonomie zurückzu-

\footnotetext{
${ }^{40}$ In diesem Fall wird die Strategie der „Habitusanpassung“ (cf. Steinhoff 2003) verwendet (cf. Roncoroni 2010: 198).
} 
führen, sondern sie sind zu einem erwarteten Kontextualisierungshinweis geworden (cf. Dürscheid 2003: 333). SMS-Schreiber könnten eventuell dazu tendieren, in ihrer Sprachproduktion im Allgemeinen zu wenige Artikel zu verwenden (cf. Dürscheid 2003: 340). Allerdings sind die Verhältnisse wohl komplizierter, denn artikellose NPs finden sich beispielsweise auch in umgangssprachlichen Äußerungen von Menschen, die keine SMS verschicken (cf. Dürscheid 2003: 333). Es ist durchaus möglich, dass selbst L2Lerner des Deutschen diese Tendenzen aufgreifen.

\section{$4 \quad$ Ausblick}

Die Erforschung der behandelten Fragestellung ist im Sinne der DaF-Förderung besonders sinnvoll. Die Ergebnisse der vorliegenden Studie stellen also wichtige Erkenntnisse dar, werfen aber gleichzeitig neue Fragen auf, denen in nachfolgenden Untersuchungen nachzugehen ist.

Sicherlich gilt es erstens, den deutschen L1-Artikelgebrauch empirisch weiter zu erforschen, z. B. anhand der digitalisierten IDS-Korpora für das Deutsche. Was das Sprachenpaar Italienisch-Deutsch betrifft, ist die Erforschung des Artikelgebrauchs in zweisprachigen Gebieten wie Südtirol vielversprechend, wobei das online abrufbare und durchsuchbare Korpus Südtirol von großem Nutzen sein dürfte.

Zweitens kann die Untersuchung von Übersetzungen besonders erhellend sein.

Drittens gebührt Sprachvergleichen zwischen Deutsch und artikellosen Sprachen wie den slawischen oder dem Persischen besondere Aufmerksamkeit. Diesbezüglich liegen bereits sehr gute Ressourcen vor, da sowohl Persisch, als auch die slawischen Sprachen im KoLasKorpus und die slawischen Sprachen auch in den Falko-Korpora stark vertreten sind ${ }^{41}$

Viertens soll die Lernerproduktion untersucht werden, die ausschließlich in einer nichtdeutschsprachigen Umgebung entstand; somit lässt sich die deutschsprachige Umgebung als Einflussvariable isolieren.

Neben qualitativen oder sogar quantitativen korpuslinguistischen Studien sind schließlich semantisch-pragmatisch ausgerichtete Untersuchungen denkbar, die beispielsweise eventuelle Schwierigkeiten bei der Deutung bestimmt-markierter NPs als generisch oder spezifisch erhellen.

\section{Literatur}

Andresen, Melanie/Knorr, Dagmar (2017): KoLaS - Kommentiertes Lernendenkorpus akademisches Schreiben. http://hdl.handle.net/11022/0000-0001-B732-8 [01.03.2017].

Barton, Dagmar (2016): Generische Nominalphrasen bei deutsch-französischer Zweisprachigkeit. Zur Verwendung des Definitartikels bei erwachsenen Herkunftssprechern. Dissertation, Universität Hamburg. http://ediss.sub.uni-hamburg.de/volltexte/2016/8035/pdf/ Dissertation.pdf [20.02.2017].

\footnotetext{
${ }^{41}$ Die Schwierigkeiten beim Artikelgebrauch seitens polnischer Lerner des Deutschen sind von WitwickaIwanowska (2012) empirisch herausgearbeitet worden. Ihre Arbeit liefert auch in theoretischer Sicht wichtige Hinweise zum Artikelgebrauch im Deutschen und zu seiner didaktischen Vermittlung.
} 
Barton, Dagmar/Kolb, Nadine/Kupisch, Tanja (2015): "Definite article use with generic reference in German: an empirical study". Zeitschrift für Sprachwissenschaft 34/2: 147-173.

Bisle-Müller, Hansjörg (1991): Artikelwörter im Deutschen. Semantische und pragmatische Aspekte ihrer Verwendung. Tübingen: Niemeyer. (= Linguistische Arbeiten 267).

Brinkmann, Hennig (1962): Die deutsche Sprache. Gestalt und Leistung. Düsseldorf: Pädagogischer Verlag Schwann.

Brugger, Gerhard (1993): "Generic interpretations and expletive determiners". University of Venice Working Papers in Linguistics 3: 1-30.

Chierchia, Gennaro (1998): "Reference to kinds across languages". Natural Language Semantics 6/4: 339-405.

Dudenredaktion (eds.) (2005): Duden - Die Grammatik (Duden Band 4). 7. revidierte Auflage. Mannheim etc.: Dudenverlag.

Dudenredaktion (eds.) (2009): Duden - Die Grammatik (Duden Band 4). 8. revidierte Auflage. Mannheim etc.: Dudenverlag.

Dürscheid, Christa (2003): „Syntaktische Tendenzen im heutigen Deutsch“. Zeitschrift für Germanistische Linguistik 31: 327-342.

Ehlich, Konrad (2007 [1986]): „Funktional-pragmatische Kommunikationsanalyse“. In: Ehlich, Konrad (ed.): Sprache und sprachliches Handeln. Bd. 1: Pragmatik und Sprachtheorie. Berlin, de Gruyter: 9-28.

Helbig, Gerhard/Buscha, Joachim ( $\left.{ }^{5} 2005\right)$ : Deutsche Grammatik: Ein Handbuch für den Ausländerunterricht. Berlin: Langenscheidt.

Knorr, Dagmar/Andresen, Melanie (2017): Commented Learner Corpus Academic Writing (KoLaS). Archived in Hamburger Zentrum für Sprachkorpora. Version 2.0. http://hdl.han dle.net/11022/0000-0001-B732-8 [01.03.2017].

Korpus Südtirol. Redaktion: Team Korpus Südtirol. Korpus Südtirol. Europäische Akademie Bozen. http://www.korpus-suedtirol.it/Pages/zusammenfassung.aspx [10.03.2017].

Krifka, Manfred (1995): Kinds of kind reference. Handout für die Conference on Genericity. Universität $\mathrm{zu}$ Köln. http://citeseerx.ist.psu.edu/viewdoc/download?doi=10.1.1.452.9 835\&-rep=rep1\&type=pdf [09.02.2017].

Krifka, Manfred (2004): "Bare NPs: Kind-referring, Indefinites, Both, or Neither?” Empirical Issues in Formal Syntax and Semantics 5: 111-132.

Krifka, Manfred et al. (1995): "Genericity: An Introduction". In: Carlson, Gregory N./Pelletier, Francis J. (eds.): The generic book. Chicago, University of Chicago Press: 1124.

Longobardi, Giuseppe (1994): "Reference and proper names. A theory of N-Movement in syntax and logical form". Linguistic Inquiry 25/4: 609-665.

Longobardi, Giuseppe (2001): “The structure of DPs. Some principles, parameters, and problems”. In: Baltin, Mark/Collins, Chris (eds.): The Handbook of Contemporary Syntactic Theory. Oxford, Blackwell: 562-603.

Oosterhof, Albert (2004): “Generic noun phrases in Dutch". In: Karlsson, Fred (ed.): Proceedings of the 20th Scandinavian Conference of Linguistics, Helsinki, January 7-9, 2004. Scandinavian Conference of Linguistics. Helsinki, University of Helsinki Department of General Linguistics: 1-22. 
Renzi, Lorenzo/Salvi, Giampaolo/Cardinaletti, Anna (eds.) (2001): Grande grammatica italiana di consultazione. Vol. 1: La Frase. I sintagmi nominale e preposizionale. Bologna: Il Mulino.

Reznicek, Marc et al. (2012): Das Falko-Handbuch Korpusaufbau und Annotationen Version 2.01. Humboldt-Universität zu Berlin. https://www.linguistik.huberlin.de/de/institut/professuren/korpuslinguistik/forschung/falko/FalkoHandbuchV2/ at_download/file [20.02.2017].

Riegel, Martin/Pellat, Jean-Christophe/Rioul, René (eds.) ( $\left.{ }^{4} 2011\right)$ : Grammaire méthodologique du français. Paris: Quadrige/Presses Universitaires de France.

Roncoroni, Tiziana (2010): „Beobachtungen zu funktionalen und formalen Fremdheitsmerkmalen in Hausarbeiten italophoner Studierender". In: Heller, Dorothee (ed.): Die mehrsprachige Wissenschaftskommunikation. Frankfurt a. M., Lang: 181-200.

Roncoroni, Tiziana (2011): „Hausarbeiten - Schreibanforderung an nicht muttersprachliche Studierende. Beobachtungen zur Textstrukturierung und Leserorientierung“. In: Knorr, Dagmar/Nardi, Antonella (eds.): Fremdsprachliche Textkompetenz entwickeln. Frankfurt a. M., Lang: 69-89.

Steinhoff, Torsten (2003): „Wie entwickelt sich die wissenschaftliche Textkompetenz?“. Der Deutschunterricht 3/3: 38-47.

Stollhans, Sascha (2012): „Ich trinke gerne *die Tee. Der Nullartikel aus der Perspektive französischsprachiger Deutschlerner unter besonderer Berücksichtigung generischer Ausdrücke“. Info DaF 6: 605-624.

Zamparelli, Roberto (2002): "Definite and bare kind-denoting noun phrases". In: Beyssade, Claire et al. (eds.): Romance Languages and Linguistic Theory 2000: Selected Papers from 'Going Romance 2000'. Amsterdam, John Benjamins: 305-343.

Witwicka-Iwanowska, Magdalena (2012): Artikelgebrauch im Deutschen. Eine Analyse aus der Perspektive des Polnischen. Tübingen: Narr.

Zifonun, Gisela et al. (eds.) (1997): Grammatik der deutschen Sprache. Band 1 und Band 3. Berlin/New York: de Gruyter. 